\title{
Efficient simulation using saddlepoint approximation for aggregate losses with large frequencies
}

\author{
Jae-Rin $\mathrm{Cho}^{a}$, Hyung-Tae $\mathrm{Ha}^{1, b}$ \\ ${ }^{a}$ Korea Insurance Research Institute, Korea \\ ${ }^{b}$ Department of Applied Statistics, Gachon University, Korea
}

\begin{abstract}
Aggregate claim amounts with a large claim frequency represent a major concern to automobile insurance companies. In this paper, we show that a new hybrid method to combine the analytical saddlepoint approximation and Monte Carlo simulation can be an efficient computational method. We provide numerical comparisons between the hybrid method and the usual Monte Carlo simulation.
\end{abstract}

Keywords: large claim frequency, aggregate claim amount, saddlepoint approximation, simulation

\section{Introduction}

Compounding distributions are a major concern in insurance risk models to model the aggregate claim amounts in a fixed period of time. The accurate and efficient computation for the distribution of the total aggregate loss should be a mandatory step to determine managerial issues (such as the optimal premium and reservoir) for insurance companies. Closed-form solutions of the distribution of the collective risk models are not available in most cases; however, recursive methods for certain classes such as Panjer class have been discussed to calculate exact quantities. Since the recursive methods are often extremely time consuming, computational methods such as Monte Carlo simulation have extensively been discussed, which become even more competitive due to the progression of modern computer processing power.

In this paper, we are interested in aggregate claim amounts with a large number of claims, which often occur in car insurance policies. For example, the expected number of claims is 10,000 for a car insurance company that has 0.1 million policy holders with $10 \%$ accident probability during a certain period of time. In such circumstances, even Monte Carlo simulation may not be very efficient to calculate the distributions or quantiles. Some researchers such as Embrechts et al. (1985), Gatto (2010), and Jensen (1991), utilized analytical approximation methods such as saddlepoint approximation to solve this inefficiency. Whereas the saddlepoint approximation is known to be computationally efficient in addition to providing very accurate tail probabilities, calculation for some distributional quantities such as quantiles still remain challenging because the inversion of the saddlepoint distribution approximation is failed in most cases.

We introduce a hybrid computational method to combine the benefits from two different methods of Monte Carlo simulation and saddlepoint approximations to achieve both quantile computation

\footnotetext{
${ }^{1}$ Corresponding author: Department of Applied Statistics, Gachon University, 1342 Sungnamdae-ro, Sujung-gu, Sungnam-ci, Kyunggi-do 13120, Korea. E-mail: htha@gachon.ac.kr
}

Published 31 January 2016 / journal homepage: http://csam.or.kr (c) 2016 The Korean Statistical Society, and Korean International Statistical Society. All rights reserved. 
and computational efficiency for aggregate insurance losses with large frequencies that were originally discussed in McLeish (2014). Daniels (1954) and Lugannani and Rice (1980) versions of the saddlepoint approximation will be utilized as a scheme to determine acceptance-rejection regions. Numerical comparisons in Section 3 admit that the hybrid method can be a viable solution to efficiently provide quantiles of compounding distributions with large frequencies.

\section{Simulation using saddlepoint approximation}

We first introduce a collective risk model. Let $Z$ be the total claim amounts occurred in an insurance portfolio within a given period of time, which is a sum of a stochastically determined number $N(t)$ of independent random variables $X_{i}$ with distribution function $F_{i}$. The collective risk model can be expressed as

$$
Z=X_{1}+\cdots+X_{N(t)},
$$

where claim frequency $N(t)$ is a discrete positive random variable with $p_{n}=\operatorname{Pr}(N(t)=k), k=$ $0,1, \ldots$ and $X_{i}$ is a continuous random variable with probability density function $f_{i}$. There is a finite probability of no loss occurring over the considered time period if $N(t)=0$ is allowed, i.e. $\operatorname{Pr}(N(t)=$ $0)=\operatorname{Pr}(Z=0) . N(t)$ and the single of random variables $X_{i}$ are independent for all $i$.

The distribution of the aggregate loss, denoted as $H(\ell)$, can be expressed in terms of convolution formula as

$$
\begin{aligned}
H(\ell) & =\operatorname{Pr}(Z \leq \ell)=\sum_{k=0}^{\infty} \operatorname{Pr}(Z \leq \ell \mid N(t)=k) \operatorname{Pr}(N(t)=k) \\
& =\sum_{k=0}^{\infty} p_{k} F^{(k) *}(\ell),
\end{aligned}
$$

where $F^{(k) *}(\ell)=\operatorname{Pr}\left(X_{1}+\cdots+X_{k} \leq \ell\right)$ is the $k^{\text {th }}$-convolution formula of $F(\cdot)$ calculated recursively from $(k-1)^{t h}$-convolution as

$$
F^{(k) *}(\ell)=\int_{0}^{\ell} F^{(k-1) *}(\ell-x) f(x) d x
$$

with

$$
F^{(0) *}(\ell)= \begin{cases}1, & \ell \geq 0, \\ 0, & \ell<0 .\end{cases}
$$

Though the obtained formula is analytic, its direct calculation is involves many integrations, such that the computations in practice are extremely heavy. Whereas the convolution are available in closedform only in special cases, its moment generating function, denoted by $\chi(s)$, can be simply expressed as

$$
\chi(s)=\sum_{i=0}^{\infty}(\varphi(s)) p_{i}=\psi(\varphi(s)),
$$

where the moment and probability generating functions of random variables $X$ and $N(t)$ are respectively $\varphi(s)=E\left[e^{s X}\right]$, and $\psi(k)=E\left[k^{N(t)}\right]=\sum_{i=0}^{\infty} k^{i} p_{i}$. 
Once the moment generating function of an aggregate claim distribution is well defined in closed functional form, a saddlepoint approximation may be employed to provide accurate approximate density and distribution functions. On letting the cumulant generating function $\mathcal{K}(s)=\log [\chi(s)]$, for $s \in \mathcal{D}=\left(-t_{0}, t_{1}\right)$, where the domain $\mathcal{D}$ is a possibly semi- or infinite interval with $-t_{0}<0<t_{1}$. When a unique solution $(t=\hat{t})$ is obtained from equating $\mathcal{K}^{(1)}(s)=x$ over the support, where $\mathcal{K}^{(i)}(s)$ is the $i^{\text {th }}$ derivative of the cumulant generating function, Daniels (1954) version of the saddlepoint density approximation (SPA) is

$$
f_{S P}(x)=\left(2 \pi \mathcal{K}^{(2)}(\hat{t})\right)^{-\frac{1}{2}} \exp (\mathcal{K}(\hat{t})-x \hat{t}) .
$$

Normalization of the saddlepoint density approximation is required because the saddlepoint density approximant does not sum to unity. The normalized saddlepoint density approximant is often more accurate than the unnormalized saddlepoint density approximant. On denoting the normalizing factor by $\eta=\left(\int_{0}^{\infty} f_{S P}(x) d x\right)^{-1}$, the normalized saddlepoint density approximant (N-SPA) can be obtained as

$$
f_{N S P}(x)=\eta f_{S P}(x) \text {. }
$$

The Lugannani and Rice (1980) version of saddlepoint distribution approximation can directly approximate tail probabilities $\operatorname{Pr}(Z \geq v)$, that is,

$$
\operatorname{Pr}(Z \geq v) \approx 1-\Phi(\hat{w})+\phi(\hat{w})\left(\frac{1}{\hat{u}}-\frac{1}{\hat{w}}\right),
$$

where $\hat{w}=\sqrt{2(\hat{s} v-\mathcal{K}(\hat{s}))} \operatorname{sgn}(\hat{s}), \hat{u}=\hat{s} \sqrt{\mathcal{K}^{(2)}(\hat{s})}, \operatorname{sgn}(\hat{s})= \pm 1,0$ if $\hat{s}$ is positive, negative, or zero, and $\phi(\cdot)$ is the standard normal density function and $\Phi(\cdot)$ is the corresponding cumulative distribution function. This Lugannani and Rice formula is undefined if $\hat{u}=\hat{w}=0$, which occurs when $v=E(Z)$ or $\hat{s}=0$. The approximation in such case should be

$$
\operatorname{Pr}(Z \geq v) \approx \frac{1}{2}-(2 \pi)^{-\frac{1}{2}}\left(\frac{1}{6} \mathcal{K}^{(3)}(0)\left(\mathcal{K}^{(2)}(0)\right)^{-\frac{3}{2}}-\frac{1}{2}\left(\mathcal{K}^{(2)}(0)\right)^{-\frac{1}{2}}\right) .
$$

Now, we let $g(x)$ be the inverse function of $\mathcal{K}^{(1)}(s)=x$ so that the solution above is $\hat{s}=\mathcal{K}^{(1)^{-1}}(x)=$ $g(x)$. Consider a transformed random variable $Y=g(X)$. From the transformation of variable techniques, the saddlepoint density and distribution approximants of $Y$ are respectively

and

$$
\begin{aligned}
f_{Y}(y) & =f_{S P}(x)\left|\frac{\partial x}{\partial y}\right| \\
& =(2 \pi)^{-\frac{1}{2}}\left(\mathcal{K}^{(2)}(y)\right)^{\frac{1}{2}} \exp \left(\mathcal{K}(y)-\mathcal{K}^{(1)}(y) y\right),
\end{aligned}
$$

$$
\begin{aligned}
\operatorname{Pr}(Y \leq v) & =\operatorname{Pr}\left(X \leq \mathcal{K}^{(1)}(v)\right) \\
& =\Phi(w(y))-\phi(w(y))\left(\frac{1}{u(y)}-\frac{1}{w(y)}\right),
\end{aligned}
$$

where $w(y)=\sqrt{2(y v-\mathcal{K}(y))} \operatorname{sgn}(y)$ and $u(y)=y \sqrt{\mathcal{K}^{(2)}(y)}$. We use acceptance-rejection to generate random numbers under the saddlepoint density (2.3). We require a simple function to cover the saddlepoint density of $Y$, which can usually be a constant multiple of a typical continuous probability density function, from which random numbers can easily be generated. We call the covering simple function dominating density. Then, the area of saddlepoint density and the area between dominating density and saddlepoint density are used as acceptance and rejection regions, respectively. 


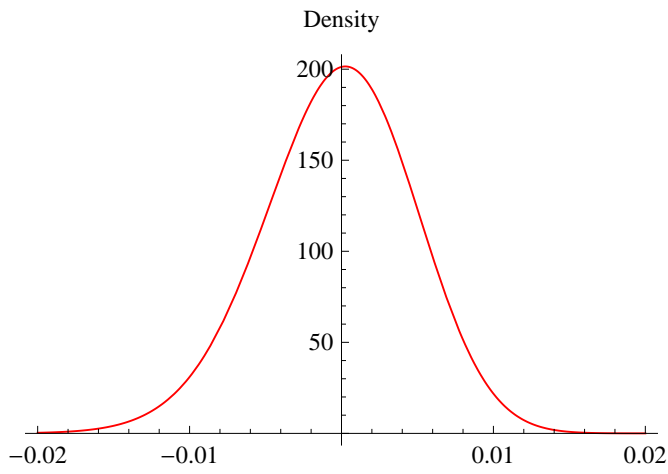

(a) $\lambda_{t}=100, \theta=1 / 4$ and $\alpha=80$

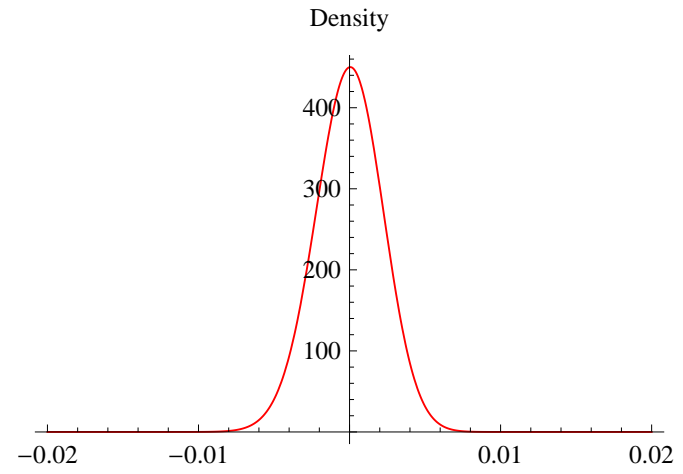

(b) $\lambda_{t}=500, \theta=1 / 4$ and $\alpha=80$

Figure 1: The saddlepoint density function for $T=g(Y)$.

\section{Aggregate losses with large frequencies: numerical examples}

Suppose we want to simulate values of the random variables $Z=\sum_{i=0}^{N(t)} X_{i}$ where the frequency random variable $N(t)$ follows a Poisson distribution with parameter $\lambda_{t}$ and $X$ follows an independent gamma random variable with parameters $\theta$ and $\alpha$. Then

$$
\chi(s)=\sum_{i=0}^{\infty}(\varphi(s))^{i} \frac{e^{-\lambda_{t}} \lambda_{t}^{i}}{i !}=\exp \left(\lambda_{t} \varphi(s)-\lambda_{t}\right),
$$

where $\varphi(s)=(1-\theta s)^{-\alpha}$. Therefore, the cumulant generating function and its first and second derivatives of $Z$ are respectively

$$
\mathcal{K}(s)=\lambda_{t}(1-\theta s)^{-\alpha}-\lambda_{t}, \quad \mathcal{K}^{(1)}(s)=\alpha \theta \lambda_{t}(1-\theta s)^{-\alpha-1} \quad \text { and } \quad \mathcal{K}^{(2)}(s)=\alpha(\alpha+1) \theta^{2} \lambda_{t}(1-\theta s)^{-\alpha-2} .
$$

Fortunately, the exact expression for the saddlepoint obtained by equating the equation $\mathcal{K}^{(1)}(s)=x$ can be determined as $\hat{s}=\theta^{-1}\left[1-\left\{x /\left(\alpha \theta \lambda_{t}\right)\right\}^{-1 /(\alpha+1)}\right]$, that is, the inverse function $g(x)=\theta^{-1}[1-$ $\left.\left\{x /\left(\alpha \theta \lambda_{t}\right)\right\}^{-1 /(\alpha+1)}\right]$. Therefore, we consider a new random variable $\mathcal{K}^{(1)}(Y)=X$, that is, $Y=\theta^{-1}[1-$ $\left\{X /\left(\alpha \theta \lambda_{t}\right)^{-1 /(\alpha+1)}\right]$. The important step for this efficient simulation using saddlepoint approximation is to determine the dominating density for acceptance-rejection. We utilize constant multiples of two simple distributions, uniform and normal distributions, to cover most of the range of the probability density function of $Y$. It should be noted that the two simple cannot cover the entire range of the saddlepoint density function of $Y$ since the saddlepoint density of $Y$ has heavy tails. But, in practice, if probability of saddlepoint density corresponding to the covered range is close to unity, that is, dominating density covers a wide range of the saddlepoint density, the random number generation may be minimally influenced from the uncovered tail.

Figure 1 shows the saddlepoint densities; the left panel for the case of $\lambda_{t}=100, \theta=1 / 4$ and $\alpha=80$, and the right panel for the case of $\lambda_{t}=500, \theta=1 / 4$ and $\alpha=80$. Obviously, its variance becomes smaller when $\lambda_{t}$ increases. Figure 2 shows the dominating function for $Y=g(X)$ with $\lambda_{t}=500, \theta=1 / 4$ and $\alpha=80$; the left panel for the case of uniform distribution with a support $(-0.02,0.02)$ and the left panel for the case of normal distribution with mean 0 and standard deviation 0.03. The multiplicative constants are 450.005 and 3.6 for uniform and normal dominating densities, respectively. 


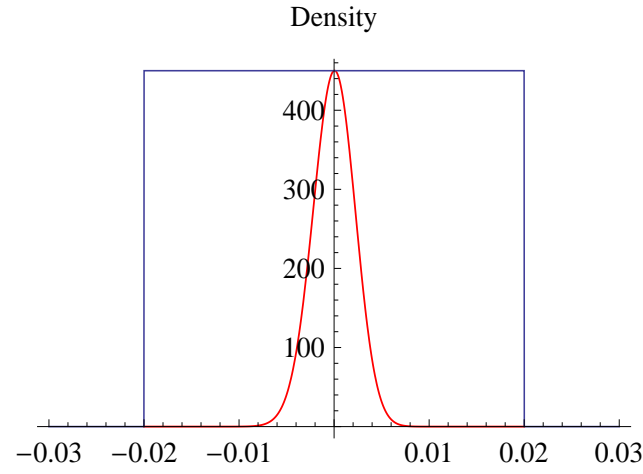

(a) $450.005 \times$ Uniform distribution with a support $(-0.02,0.02)$

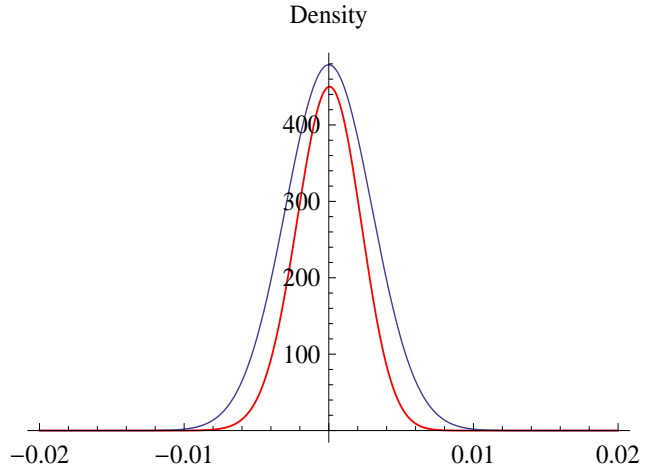

(b) $3.6 \times$ Normal distribution with mean 0 and standard deviation 0.03

Figure 2: The dominating function for $T=g(Y)$ with $\lambda_{t}=500, \theta=1 / 4$ and $\alpha=80$.

Table 1: Computation time in seconds

\begin{tabular}{crrrrr}
\hline \hline & Case & \multicolumn{3}{c}{$\mathrm{RN}$} \\
\cline { 3 - 6 } & & 1,000 & 10,000 & 100,000 & $1,000,000$ \\
\hline \multirow{2}{*}{ Computation time } & $\lambda_{t}=100$ & 2.652 & 4.089 & 17.596 & 153.396 \\
with uniform & $\lambda_{t}=500$ & 2.682 & 4.882 & 25.459 & 231.208 \\
dominating function & $\lambda_{t}=1,000$ & 2.777 & 4.682 & 25.068 & 232.347 \\
& $\lambda_{t}=10,000$ & 2.791 & 4.805 & 25.692 & 241.958 \\
\hline \multirow{2}{*}{ Computation time } & $\lambda_{t}=100$ & 2.761 & 3.759 & 18.751 & 121.946 \\
with normal & $\lambda_{t}=500$ & 2.605 & 3.589 & 14.822 & 110.276 \\
dominating function & $\lambda_{t}=1,000$ & 2.543 & 3.510 & 13.042 & 109.139 \\
& $\lambda_{t}=10,000$ & 2.497 & 3.555 & 13.073 & 110.386 \\
\hline \multirow{2}{*}{ Computation time } & $\lambda_{t}=100$ & 6.150 & 39.050 & 313.080 & 3418.530 \\
using Monte & $\lambda_{t}=500$ & 19.640 & 154.062 & 1743.420 & 14692.600 \\
Carlo simulation & $\lambda_{t}=1,000$ & 36.456 & 334.592 & 2853.410 & 29493.000 \\
& $\lambda_{t}=10,000$ & 313.594 & 3341.010 & 28844.800 & 284931.000 \\
\hline \hline
\end{tabular}

RN = Random Numbers.

Now, we compare the efficiency and accuracy of the proposed method and the usual Monte Carlo simulation. Consider various cases of large claims $\lambda_{t}=100,500,1,000,10,000$ when $\theta=1 / 4$ and $\alpha=80$. Table 1 compares the computational times between the proposed methods with uniform and normal dominating functions, and Monte Carlo simulation. Both proposed methods using uniform and normal dominating functions are extremely fast with comparison to the usual Monte Carlo simulation (Table 1). For instance, in order to generate 0.1 million numbers when $\lambda_{t}=10,000$, the proposed method with uniform and normal dominating functions spends 232.347 and 109.139 seconds, whereas the Monte Carlo Simulation requires 29493. However, the proposed methods, using uniform and normal dominating functions, are more efficient than Monte Carlo Simulation by 127 and 270 times, respectively. The random number generation from uniform distribution is faster than normal distribution; however, the proposed method using normal dominating functions is more efficient than one using uniform dominating functions because the normal dominating function significantly reduces the rejection region. Table 2 shows the quantiles for the various cases. It is seen that those three methods provide similar estimates for the $90 \%, 95 \%$ and $99 \%$ quantiles. RN represents the number of generated random numbers. The numerical comparison was conducted on a personal computer 
Table 2: Accuracy with uniform and normal functions and simulation

\begin{tabular}{|c|c|c|c|c|c|}
\hline \multirow{2}{*}{$\mathrm{RN}$} & \multirow{2}{*}{ Cases } & \multicolumn{4}{|c|}{$\lambda_{t}$} \\
\hline & & 100 & 500 & 1,000 & 10,000 \\
\hline \multirow{9}{*}{1,000} & \multirow{3}{*}{$90 \%$} & 2257.43 & 10474.9 & 20627.5 & 199312 \\
\hline & & 2285.81 & 10547.5 & 20773.2 & 202485 \\
\hline & & 2251.04 & 10542.1 & 20844.6 & 202534 \\
\hline & \multirow{3}{*}{$95 \%$} & 2318.46 & 10660.7 & 20876.1 & 201042 \\
\hline & & 2353.89 & 10770.0 & 21032.9 & 203249 \\
\hline & & 2312.60 & 10750.6 & 21079.4 & 203304 \\
\hline & \multirow{3}{*}{$99 \%$} & 2483.62 & 11059.9 & 21240.3 & 203334 \\
\hline & & 2506.41 & 11107.1 & 21454.8 & 204462 \\
\hline & & 2495.39 & 11028.3 & 21530.7 & 205243 \\
\hline \multirow{9}{*}{10,000} & \multirow{3}{*}{$90 \%$} & 2258.01 & 10489.8 & 20543.8 & 199447 \\
\hline & & 2261.31 & 10573.2 & 20825.7 & 202659 \\
\hline & & 2262.98 & 10575.9 & 202450 & 202560 \\
\hline & \multirow{3}{*}{$95 \%$} & 2331.34 & 10666.3 & 20823.5 & 201001 \\
\hline & & 2337.07 & 10743.2 & 21064.4 & 203420 \\
\hline & & 2349.65 & 10731.4 & 203107 & 203275 \\
\hline & \multirow{3}{*}{$99 \%$} & 2479.65 & 10993.3 & 21331.2 & 202952 \\
\hline & & 2474.01 & 11047.4 & 21514.3 & 204615 \\
\hline & & 2493.56 & 11048.8 & 204376 & 204759 \\
\hline \multirow{9}{*}{100,000} & \multirow{3}{*}{$90 \%$} & 2259.20 & 10485.0 & 20530.1 & 199282 \\
\hline & & 2258.09 & 10577.6 & 20814.8 & 202572 \\
\hline & & 2259.02 & 10579.6 & 20823.1 & 202567 \\
\hline & \multirow{3}{*}{$95 \%$} & 2336.90 & 10672.0 & 20815.7 & 200934 \\
\hline & & 2334.76 & 10744.5 & 21046.5 & 203312 \\
\hline & & 2335.81 & 10747.1 & 21055.1 & 203313 \\
\hline & \multirow{3}{*}{$99 \%$} & 2485.30 & 10998.0 & 21319.7 & 203066 \\
\hline & & 2476.82 & 11047.6 & 21491.5 & 204678 \\
\hline & & 2480.68 & 11060.4 & 21483.3 & 203938 \\
\hline \multirow{9}{*}{$1,000,000$} & \multirow{3}{*}{$90 \%$} & 2260.56 & 10478.6 & 20530.3 & 199274 \\
\hline & & 2260.07 & 10578.6 & 20818.4 & 202579 \\
\hline & & 2260.54 & 10759.4 & 20817.9 & 202577 \\
\hline & \multirow{3}{*}{$95 \%$} & 2337.20 & 10662.6 & 20814.7 & 200937 \\
\hline & & 2336.59 & 10746.7 & 21052.6 & 203306 \\
\hline & & 2336.95 & 10746.5 & 21052.1 & 203307 \\
\hline & \multirow{3}{*}{$99 \%$} & 2482.09 & 10998.5 & 21315.8 & 203060 \\
\hline & & 2482.51 & 11061.3 & 21494.0 & 204687 \\
\hline & & 2482.65 & 11062.6 & 21496.4 & 203940 \\
\hline
\end{tabular}

RN = Random Numbers.

with a Core TM i5-4210M CPU 2.6 GHZ.

\section{Concluding remarks}

Saddlepoint approximation was utilized to construct acceptance-rejection algorithm to generate random numbers for compounding sums. The choice of dominating density shall be carefully decided because the performance of the proposed simulation depends on the simplicity and closeness of the dominating density to the saddlepoint approximation of the aggregate claim distribution. In addition, simple dominating density for acceptance-rejection such as uniform or normal distributions can often provide accurate quantiles of compounding sums that are extremely efficient in computation. The proposed hybrid methods can be considered as a viable alternative to obtain the probabilistic quantities of compounding sums with large claim frequencies. It will be interesting to study the analytical and computational properties of an appropriate dominating density under various future circumstances. 


\section{Acknowledgements}

This research was supported by the Gachon University research fund of 2014 (GCU-2014-0172). The authors wish to express sincere thanks to the editor and two anonymous referees.

\section{References}

Daniels HE (1954). Saddlepoint approximations in statistics, Annals of Mathematical Statistics, 25, 631-650.

Embrechts P, Jensen JL, Maejima M, and Teugels JL (1985). Approximations for compound Poisson and Plya processes, Advances in Applied Probability, 17, 623-637.

Gatto R (2010). A saddlepoint approximation to the distribution of inhomogeneous discounted compound Poisson processes, Methodology and Computing in Applied Probability, 12, 533-551.

Jensen JL (1991). Saddlepoint approximations to the distribution of the total claim amount in some recent risk models, Scandinavian Actuarial Journal, 1991, 154-168.

Lugannani R and Rice SO (1980). Saddlepoint approximation for the distribution of the sum of independent random variables, Advances in Applied Probability, 12, 475-490.

McLeish D (2014). Simulating random variables using moment-generating functions and the saddlepoint approximation, Journal of Statistical Computation and Simulation, 84, 324-334. 